\title{
Effect of Propeller Suction on Wake
}

\author{
Tetsuo Nagamatsu,* Member \\ Takao Sasajima,* Member
}

\begin{abstract}
Summary
To examine the relation between effective wake fraction and propeller thrust loading coefficient, the contraction of flow in front of a propeller due to propeller suction was studied by use of the flow visualization method. It was shown that the rate of the contraction depends mainly upon the propeller thrust loading coefficient and is nearly estimated by the momentum theory.

The nominal mean wake was calculated by taking the effect of the contraction of flow into consideration. The remarkable decrease of effective wake fraction with increase of propeller thrust loading coefficient which is normally seen in model test results of full form ship can be explained by the effect of propeller suction.
\end{abstract}

\section{Introduction}

In many cases of full ship models, disagreement exists between nominal mean wake and effective wake fraction obtained from self-propulsion tests. Considering the fact that disagreement becomes. larger with increase of fullness of ship models, it can be easily imagined that the effect of propeller suction strongly affects the flow field in front of a propeller, as propeller thrust loading coefficient becomes relatively large for such type of ships.

Nominal mean wake is obtained by averaging the wake over a propeller disc, the distribution of which is measured at the propeller plane without a propeller. It may be more reasonable to take the effect of propeller suction into account in the calculation of nominal mean wake.

The stream line of the contraction of flow in front of a propeller can be calculated by actuator disc theory. Up to the present, however, there are few experimental data relating to the shape of the contraction of flow.

Thus, following studies were carried out by the authors in Nagasaki Experimental Tank of MHI.

At first, flow visualization tests were carried out to examine the contraction of flow in front of a propeller and test results were compared with those of calculation.

Then, based on the examination above-mentioned, nominal mean wake was calculated, taking the effect of the contraction of flow in front of a propeller into consideration, and was compared with the effective wake fraction.

\section{Flow visualization tests}

To visualize the contraction of flow in front of a propeller, an air injection method was applied to the tests in the cavitation tunnel. The air injector, to which a syringe was applied, was set at the position about a diameter before the propeller. The injector could be moved up and down in the vertical center plane of the measuring section.

Air bubbles were continuously injected into the tunnel, where the static pressure was kept slightly

* Nagasaki Technical Institute, Mitsubishi Heavy Industries, Ltd. 
lower than the atmospheric pressure. The trace of air bubbles was measured by bar gauges, which were inserted from the tunnel wall to touch flowing air bubbles.

Fig. 1 shows an example of the air injection tests, in which flowing air bubbles just pass through the propeller tip under the effect of propeller suction. As air bubbles flow also under the effect of

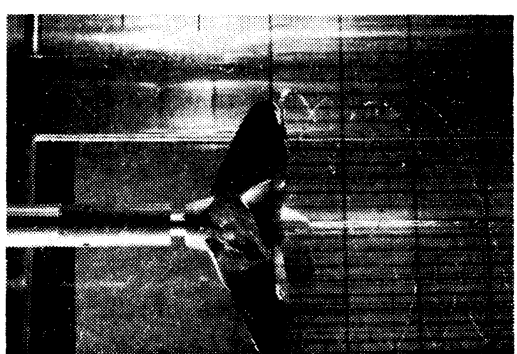

Fig. 1 Example of flowing air bubbles under the effect of propeller suction

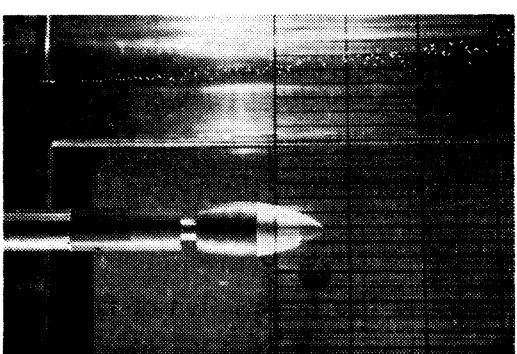

Fig. 2 Example of flowing air bubbles without the effect of propeller suction

buoyancy as shown in Fig. 2, the trace of flowing air bubbles must be corrected for this effect. Furthermore, as the effect buoyancy on air bubbles changes with the static pressure, i.e. with the vertical position in the measuring section, measurements were conducted in two ways.

Case 1: Air bubbles were injected in the upper side of the tunnel.

Case 2: Air bubbles were injected in the lower side of the tunnel.

In both cases, the trace of air bubbles under the effect of propeller suction was corrected for their buoyancy effect by use of the results measured without a propeller, as illustrated in Fig. 3.

Fig. 4 shows the shape of the contraction of flow thus obtained. A simple calculation was carried

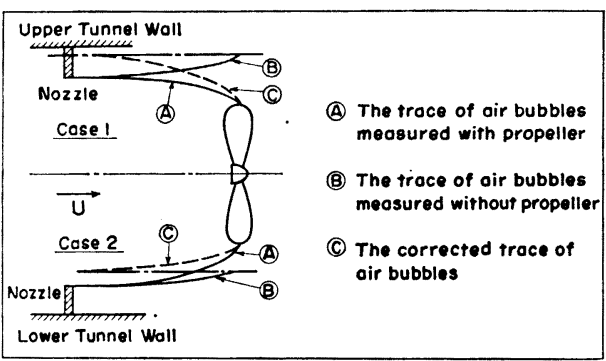

Fig. 3 Method of correction of buoyancy effect

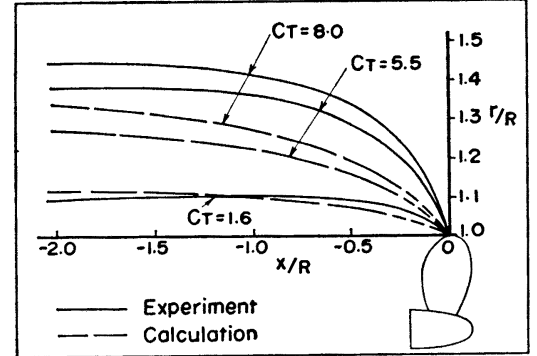

Fig. 4 Shape of the contraction of flow before the propeller

out by use of actuator disc theory ${ }^{1)}$, in which the propeller disc is represented by uniform sink distribution. In this case, the strength of $\operatorname{sink} q$ is given as follows.

$$
q / U=-1+\sqrt{1+C_{T}}
$$

where, $\quad C_{T}=T / \frac{1}{2} \pi \rho U^{2} R^{2}:$ Propeller thrust loading coefficient

$U$ : Advance speed of propeller

$R$ : Propeller radius

$T$ : Propeller thrust

The stream line which passes through the propeller tip can not be calculated by use of the sink disc above-mentioned, since the radial velocity component becomes infinite at the propeller tip. So the shape of the contraction of flow was substituted by a stream line which passes through the po- 
sition $0.01^{R}$ before the propeller tip.

It is noticed that the shape of the contraction measured just before the propeller changes more sharply than that calculated. This may be caused by the fact that the thrust of the propeller is distributed larger in the outer part of propeller disc, while uniform thrust distribution is assumed in the calculation ${ }^{2}$.

It is worth noticing that the flow measured at the position about a diameter before the propeller, which contracts to the propeller disc, is almost parallel to uniform flow and that the radius of the cross section of this flow $R_{0}$ can be approximated by formula (2), which is obtained far before the propeller by momentum theory, as shown in Fig. 5 .

$$
\left(R_{0} / R\right)^{2}=\frac{1}{2}\left(1+\sqrt{1+C_{T}}\right)
$$

The shape of the contraction of flow in wake was also examined. Two types of the concentric wake distribution generated by wire meshes were used here. The radial distribution of these wakes are shown in Fig. 6. The radius of the cross section of the flow which contracts to the propeller disc

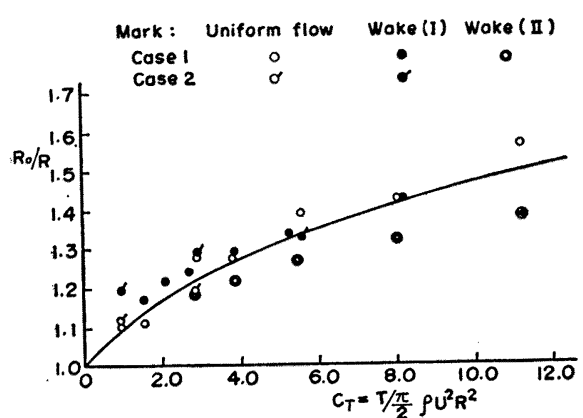

Fig. 5 Relation between the contraction of flow and propeller thrust loading coefficient

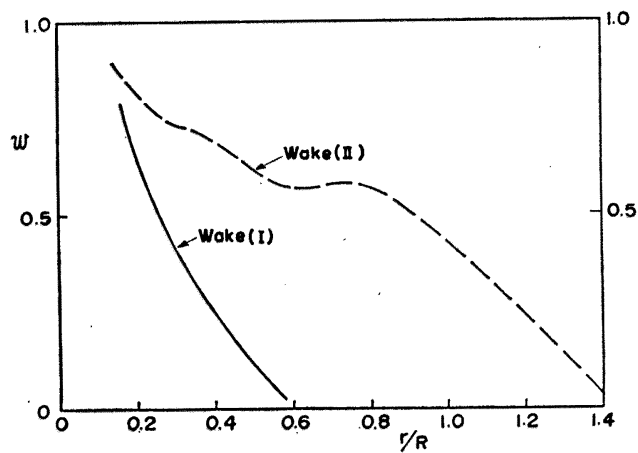

Fig. 6 Radial distribution of wake generated by concentric wire meshes

is measured at the position about a diameter before the propeller, as shown in Fig. 5. In wake (I), the radius is quite the same as that in uniform flow, since the wake is distributed only within the inner part of the propeller disc. But in wake (II), the radius is a little smaller than that in uniform flow.

This may come from the difference of the thrust distribution of the propeller in these wakes, especially near the propeller tip.

But in the following study, formula (2) is used to estimate the radius of the cross section of flow far before the propeller, as the first approximation.

\section{Effect of the contraction of flow on wake}

Nominal mean wake is usually obtained by averaging the wake over the propeller disc, the distribution of which is measured at the propeller plane without propeller. However, in the case of full form ships, for which the propeller thrust loading coefficient is considerably large, it seems more reasonable to include the effect of propeller suction in the calculation of nominal mean wake.

Imagine a wake distribution, which is quite the same as that measured at the propeller plane without a propeller, at the upstream position where the propeller suction does not affect. Due to propeller suction, a cross section of flow, in which the imagined wake is contained far before the propeller, converges into the propeller disc along the contraction of flow. Thus, if the imagined wake 
is averaged over the cross section of the flow mentioned above, the effect of propeller suction can be included in the calculation of nominal mean wake.

According to the flow visualization test results, it was shown that the radius of the cross section of the flow far before the propeller, which contracts to the propeller disc, can be approximated by one derived from the momentum theory. Thus an attempt was made to calculate nominal mean wake by averaging the wake distribution measured at the propeller plane over the cross section, the radius of which is estimated by formula (2). Then the nominal volume mean wake is given, as follows.
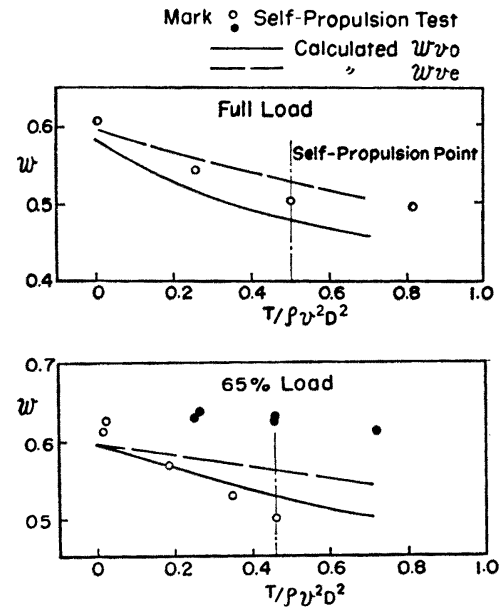

Fig. 7 Effect of propeller thrust loading coefficient on effective wake fraction for a full ship model

$$
w_{v}=\frac{\int_{x_{b}}^{1} d x \int_{0}^{2 \pi} w(x, \theta) f(x) x d \theta}{\int_{x_{b}}^{1} d x \int_{0}^{2 \pi} f(x) x d \theta}
$$

where,

$$
x=r / R_{0}, \quad x_{b}=R_{b o s s} / R_{0}
$$

$w(x, \theta):$ wake distribution

$f(x)$ : weight function

Here, the following two types of weight function were applied, referring to the propeller thrust distribution.

(1) $f(x)=$ constant: $w_{v}$ is denoted as $w_{v 0}$.

(2) $f(x)=x(1-x): w_{v}$ is denoted as $w_{v e}$.

The propeller thrust loading coefficient $C_{T}$ is expressed as. follows.

$$
C_{T}=\frac{8}{\pi\left(1-w_{v}\right)^{2}} \frac{T}{\rho V^{2} D^{2}}
$$

where, $\quad v$ : Speed of ship model

$D:$ Propeller diameter $(=2 R)$

Fig. 7 shows the results calculated by using formula (3) and (4), in comparison with self-propulsion. test results for a full ship model. Except the test results for $65 \%$ load condition marked by $\bullet$ in the
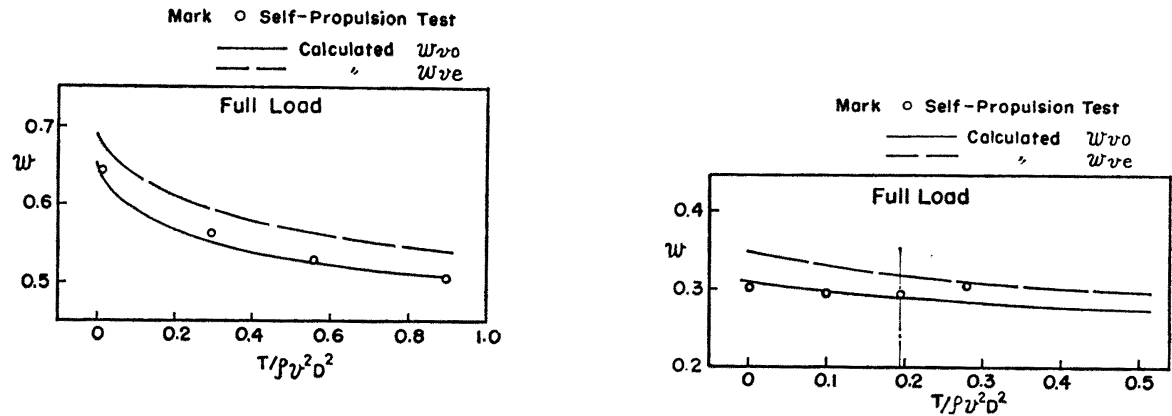

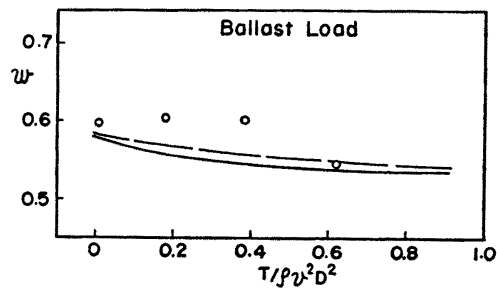

Fig. 8 Effect of propeller thrust loading coefficient on effective wake fraction for a tanker model

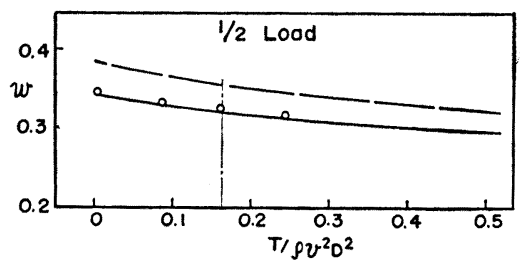

Fig. 9 Effect of propeller thrust loading coefficient on effective wake fraction: for a cargo liner model 
figure, calculated results agree well with test results.

Other examples of the calculation for a tanker model and a cargo liner model are shown in Figs. 8 and 9 respectively. Close agreement between calculation and experiment is obtained for both models. The radial distribution of the circumferential mean of the nominal wake for three ship models

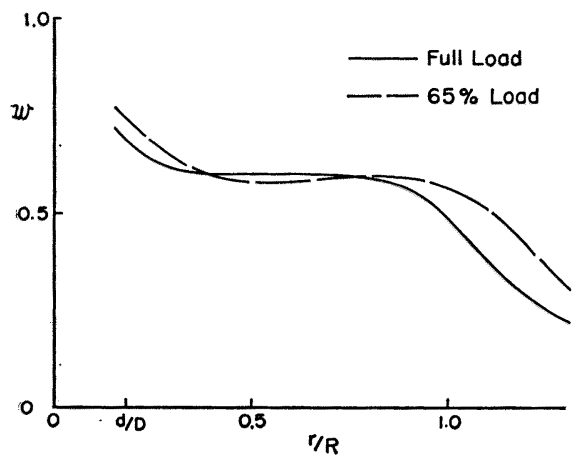

Fig. 10 Radial distribution of circumferential mean wake for a full ship model

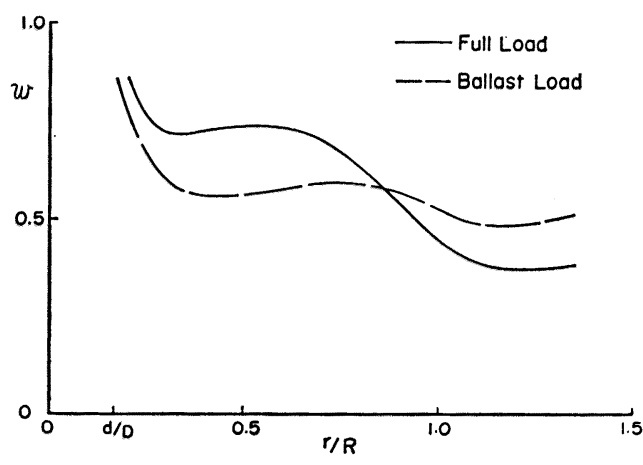

Fig. 11 Radial distribution of circumferential mean wake for a tanker model

mentioned above are shown in Figs. 10, 11 and 12 respectively. The change of the effective wake with the propeller thrust loading coefficient $T / \rho V^{2} D^{2}$ is explained as follows. In the case of the cargo liner model, not only the value of wake but also its change is small near the propeller tip, as shown in Fig. 12. Thus the effect of the contraction of flow on nominal mean wake is small, even if the radius of the averaging disc is extended to $R_{0}$. On the other hand, in the case of full ship models, as tankers, the high wake zone extends up to 0.6 -0.7 radius of the propeller and then decreases steeply, as shown in Fig. 10 for both full load and

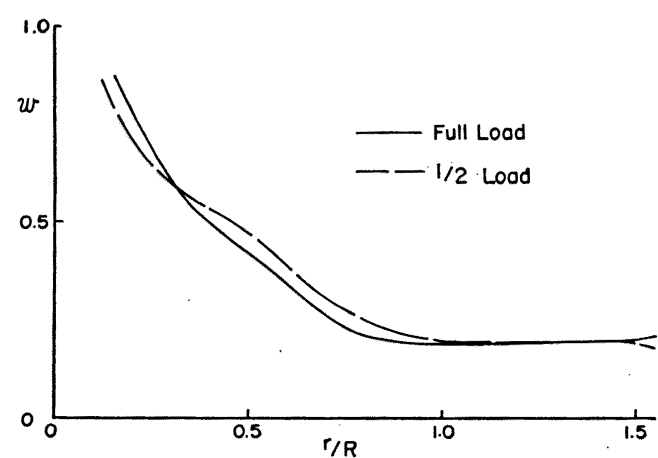

Fig. 12 Radial distribution of circumferential mean wake for a cargo liner model 65\% load conditions and in Fig. 11 for full load condition. As the propeller thrust loading coefficient for these types of ship is considerably large in comparison with that for cargo liner ships, the nominal mean wake decreases steeply with increase of the radius of averaging disc, i.e. with increase of the propeller thrust loading coefficient. But, as for the ballast load condition of a tanker model in Fig. 11 , the nominal mean wake does not change so much with the propeller thrust loading coefficient, since the change of wake near the propeller tip is rather small.

Thus it is concluded that the change of effective wake fraction due to propeller thrust loading coefficient for full ship models can be explained, in the same way, by the contraction of flow due to propeller suction, as for cargo liner models.

There remains a case, however, in which the change of effective wake fraction due to propeller thrust loading coefficient can not be explained by the effect of the contraction of flow, for instance the case marked by $\bullet$ in Fig. 7.

Concerning the unstable phenomena observed in self-propulsion tests, the flow pattern behind a ship model is classified in three types from the relation between the effective wake fraction and the propeller thrust loading coefficient. From the viewpoint of flow pattern at the stern, test results for full :ship models shown in Figs. 7 and 8 are said to correspond to " $F$ " type except the test results for $65 \%$ 
load condition marked by $\bullet$ in Fig. 7, which corresponds to " $\mathrm{S}$ " type. On the other hand, the flow type for cargo liner models is usually known as " $\mathrm{N}$ " type.

Thus it can be said that so called " $F$ " type flow is classified in " $N$ " type flow in view of the effect of propeller suction. The flow type of "S", for instance $65 \%$ load condition of a tanker model in Fig. 7, is a different one, namely abnormal type.

Considering the good agreement obtained between the effective wake fraction and the nominal mean wake in which the effect of propeller suction is included, it may be more reasonable to take the effect of the contraction of flow into account in the calculation of the propeller exciting forces or in the estimation of cavitation characteristics of a propeller. Such an attempt has already been made by A.E. Raestad ${ }^{4}$ and improvements in the estimation of the bearing forces have been reported.

\section{Concluding remarks}

The effect of propeller suction on the flow field before a propeller was studied in the cavitation tunnel. The nominal mean wake was calculated by taking the effect of the contraction of flow into consideration and compared with the effective wake fraction obtained from self-propulsion tests. The following remarks were obtained as conclusions.

(1) The remarkable change of effective wake fraction due to propeller thrust loading coefficient, obtained from self-propulsion tests, can be explained by the effect of propeller suction on the flow field before the propeller for ship models with usual stern flow pattern.

But there remains a case, where the flow condition is considerably different from the estimations, which includes the effect of propeller suction. This type of flow corresponds to so called " $\mathrm{S}$ " type.

(2) The shape of the contraction of flow measured just before a propeller in uniform flow is steeper than that estimated by simple actuator disc theory. This may be caused by the difference of the propeller thrust load distribution, since uniform thrust load distribution is assumed in the actuator disc theory.

But the formula (2) can be used to estimate the rate of the contraction of flow far before the pro. peller both in uniform flow and in wake as the first approximation.

(3) Further study is necessary for estimating the contraction of flow before a propeller in wake more accurately.

\section{Acknowledgement}

The authors wish to express their hearty appreciation to Dr. K. Watanabe, Vice-manager of Nagasaki Technical Institute, Mitsubishi Heavy Industries, Ltd. and to Mr. K. Tamura, Manager of Resistance and Propulsion Research Laboratory of the same Institute, for their continuing guidance and valuable discussions.

They also thank all the members of the Nagasaki Experimental Tank for their cooperation.

\section{References}

1) Küchemann and Weber: Aerodynamics of Propulsion, McGrawhill, (1953).

2) Greenberg, M.D.: Nonlinear Actuator Disc Theory, Zeitschaft für Flugwissenschaften, 20 Jahrgang, Heft 3, (1972).

3) Shpakoff, V.S. and Watanabe, K.: The Flow Pattern at the Stern of Full Ship Form, 13th ITTC Performance Committee, Appendix V, Hamburg, (1972).

4) Raestad, A.E.: Estimation of a Marine Propellers Induced Effects on the Hull Wake Field-Scale Effects on the Hull Wake Field, Det Norske Veritas, Report No. 72-3-M. 